\title{
Proton-Resistant Quantum Dots: Stability in Gastrointestinal Fluids and Implications for Oral Delivery of Nanoparticle Agents
}

\author{
Aaron M. Mohs, Hongwei Duan, Brad A. Kairdolf, Andrew M. Smith, and Shuming Nie $(\bowtie)$ \\ Departments of Biomedical Engineering and Chemistry, Emory University and Georgia Institute of Technology, 101 \\ Woodruff Circle NE, Suite 2001, Atlanta, Georgia 30322, USA \\ Received: 22 February 2009/Revised: 30 March 2009/Accepted: 1 April 2009 \\ (C) Tsinghua University Press and Springer-Verlag 2009. This article is published with open access at Springerlink.com
}

\begin{abstract}
Semiconductor quantum dots (QDs) have shown great promise as fluorescent probes for molecular, cellular and in vivo imaging. However, the fluorescence of traditional polymer-encapsulated QDs is often quenched by proton-induced etching in acidic environments. This is a major problem for applications of QDs in the gastrointestinal tract because the gastric (stomach) environment is strongly acidic $(\mathrm{pH} 1-2)$. Here we report the use of proton-resistant surface coatings to stabilize QD fluorescence under acidic conditions. Using both hyperbranched polyethylenimine (PEI) and its polyethylene glycol derivative (PEG-grafted PEI), we show that the fluorescence of core-shell CdSe/CdS/ZnS QDs is effectively protected from quenching in simulated gastric fluids. In comparison, amphiphilic lipid or polymer coatings provide no protection under similarly acidic conditions. The proton-resistant QDs are found to cause moderate membrane damage to cultured epithelial cells, but PEGylation (PEG grafting) can be used to reduce cellular toxicity and to improve nanoparticle stability.
\end{abstract}

\section{KEYWORDS}

Nanoparticle, quantum dot, oral delivery, gastrointestinal, polyethylenimine (PEI) , polyethylene glycol (PEG)

\section{Introduction}

Semiconductor quantum dots (QDs) are a new class of fluorescent labels under intensive research and development for broad applications in molecular, cellular, and in vivo imaging [1-4]. This intense interest arises from their unique electronic and optical properties, such as size- and composition-tunable fluorescence emission, large absorption cross sections, and exceptional brightness and photostability. Recent advances have led to the development of bright and stable QDs by using highly crystalline cores and a variety of surface coating materials [4]. The most common coatings include amphiphilic polymers [5, 6], amphiphilic lipids [7], hydrophilic thiols [8], and multidentate coordinating ligands $[8,9]$. Generally, the coated QDs are stable under neutral or basic conditions. However, recent research has shown that these traditional surface coatings are ineffective in protecting QDs against chemical oxidation by reactive oxygen species [10] or against proton etching under strongly acidic conditions [11]. Thus, the fluorescence of these encapsulated QDs is quickly quenched under strongly oxidative or acidic

Address correspondence to snie@emory.edu 
conditions. It is believed that small molecules or ions such as hypochlorous acid $(\mathrm{HOCl})$, hydrogen peroxide $\left(\mathrm{H}_{2} \mathrm{O}_{2}\right)$, and protons $\left(\mathrm{H}^{+}\right)$are able to diffuse across the surface coating layer, causing chemical oxidation or etching of chalcogen atoms (sulfur, selenium, or tellurium) on the QD surface [10]. This instability is a major concern when QDs are used for intracellular and in vivo imaging in which reactive oxygen species are generated during normal cellular metabolism and in inflammatory responses [12-14]. Furthermore, this quenching problem precludes the use of QDs as an oral delivery agent for applications in the highly acidic gastrointestinal environment [15].

In this paper, we report the use of "proton sponge" polymers as acid-stable surface coatings to stabilize QD fluorescence under acidic conditions. Proton sponge polymers contain a large number of acid and base groups, and are able to buffer $\mathrm{pH}$ changes by rapidly absorbing or releasing protons [16, 17]. Under strongly acidic conditions, their positive charges are believed to "repel" other protons from approaching the polymer surface. Here we show that both hyperbranched polyethylenimine (PEI) and its polyethylene glycol-grafted derivative (PEG$g$-PEI) are able to protect core-shell CdSe/CdS/ZnS QDs from fluorescence quenching in simulated gastric fluids (SGF, containing concentrated digestive enzymes at low $\mathrm{pH}$ ). We have also examined the biocompatibility of the acid-stable QDs by using Caco-2 cells, a type of epithelial colorectal adenocarcinoma that is widely used to predict the absorption rate of candidate drug compounds across the intestinal epithelial cell barrier [18]. As expected, the cationic QDs cause moderate membrane damage, but their toxic effect can be reduced by PEG grafting.

\section{Experimental}

Materials. Dipalmitoyl-sn-glycero-3phosphoethanolamine- $\mathrm{N}$-[methoxy(polyethylene glycol)-2000] (DPPE-PEG) and 1,2-dipalmitoyl-snglycero-3-phosphocholine (DPPC) were purchased from Avanti Polar Lipids, Inc. (Alabaster, AL). PEI, $M_{\mathrm{n}}=10,000 \mathrm{~g} / \mathrm{mol}$, was purchased from SigmaAldrich (St. Louis, MO). Polyethylene glycol (PEG) grafted PEI (PEI- $g$-PEG) was synthesized according to a previously reported method [19]. Pepsin (porcine gastric mucosa, 882 units $/ \mathrm{mg}$ protein) and pancreatin (porcine pancreas) were purchased from Sigma-Aldrich. Phosphate buffered saline (PBS) was purchased from Mediatech, Inc. (Herndon, VA). All other solvents and reagents were purchased from Sigma-Aldrich or Fisher Scientific (Pittsburgh, PA) and used without further purification. Water was obtained from a Millipore Direct-Q UV system and had a resistivity of 18.2 $\mathrm{M} \Omega \cdot \mathrm{cm}$ in all cases.

QD synthesis and surface modification. CdSe/CdS/ZnS core-shell QD nanocrystals were synthesized using previously reported methods $[11,20]$. The resulting QDs were purified from the crude reaction mixture containing octadecylamine and octadecene. The crude mixture was first dissolved in a minimal amount of chloroform followed by precipitation with acetone. After centrifugation, the thin film of QDs was dispersed in hexane and repeatedly extracted with methanol. After the final washing, QDs were again precipitated with acetone. The purified QD film was then dispersed in chloroform for surface modification. Two techniques were used to surface coat the QDs and transfer them into water - micellular encapsulation and ligand exchange. Lipid-PEG-coated QDs were prepared based on the procedure reported by Dubertret et al. [7]. Briefly, to QDs $(0.5 \mathrm{nmol})$ dispersed in chloroform was added lipid-PEG (1500 nmol) dissolved in chloroform. The chloroform was allowed to evaporate under vacuum and the remaining cake was dispersed in $\mathrm{H}_{2} \mathrm{O}$ with sonication. The solution was ultracentrifuged for $2 \mathrm{~h}$ at 100,000 rpm. The supernatant containing empty micelles was removed and the lipid-PEG-coated QD pellet was resuspended in $\mathrm{H}_{2} \mathrm{O}$. Aggregates were removed by filtering the QD-lipid-PEG nanoparticles through a $0.2 \mu \mathrm{m}$ filter. Using this procedure, QDs $(0.5 \mathrm{nmol})$ were alternatively coated with DPPE-PEG (600 $\mathrm{nmol})$ and DPPC (900 nmol), which was expected to increase the packing density of the hydrophobic barrier. A second class of QDs with cationic surfaces was synthesized by ligand exchange. PEI or PEI-g-PEG-coated QDs were prepared according to the literature method [19]. Briefly, either PEI or 
PEI- $g$-PEG was dissolved in chloroform and then shaken with QDs in chloroform to afford ligand exchange with the octadecylamine on the QD surface. After the ligand exchange reaction, the chloroform was removed in vacuo and the film was dispersed in $\mathrm{H}_{2} \mathrm{O}$. Excess polymer was removed by extensive dialysis. Any remaining aggregates were removed by filtering through a $0.2 \mu \mathrm{m}$ filter.

Characterization. Cationic polymer modified QDs were characterized by several different techniques. Absorbance spectra and fluorescence spectra were collected on a Shimadzu UV-2401PC spectrophotometer (Shimadzu Scientific Instruments, Columbia, MD) and FluoroMax-2 spectrofluorometer (HORIBA Jobin Yvon, Inc., Edison, NJ), respectively. The size of the nanoparticles was measured by dynamic light scattering (DLS) (Brookhaven 90plus, Brookhaven Instruments Corp., Holtsville, NY) and transmission electron microscopy (TEM) (Hitachi H-7500, Hitachi High Technologies America, Inc., Pleasanton, CA). The zeta potential of nanoparticles was measured on a Zetasizer Nano (Malvern Instruments, Ltd., Worcestershire, UK).

Gastrointestinal fluid studies. Gastrointestinal conditions were simulated for gastric fluid and for intestinal fluid based on previously reported protocols [21, 22]. SGF was prepared by dissolving pepsin in gastric control fluid $(2 \mathrm{mg} / \mathrm{mL} \mathrm{NaCl}, \mathrm{pH}$ 2.0) to a total concentration of $300 \mathrm{U} / \mathrm{mL}$. Simulated intestinal fluid (SIF) was prepared by dissolving pancreatin $(10 \mathrm{mg} / \mathrm{mL})$ in intestinal control fluid (0.05 mol/L KH $\mathrm{KHO}_{4}, \mathrm{pH}$ 6.8). For all studies, SGF and SIF were used on the same day as preparation. To test the fluorescence stability of each QD sample in SGF, the SGF $(360 \mu \mathrm{L})$ was first incubated for $5 \mathrm{~min}$ in a capped glass tube at $37^{\circ} \mathrm{C}$ on a heating block filled with sand to ensure uniform heating. QDs (40 $\mu \mathrm{L}, 1 \mu \mathrm{mol} / \mathrm{L}$ ) were added to the warm SGF and incubated for 5, 15, 30, or $60 \mathrm{~min}$. Acid etching was stopped by neutralizing the reaction mixture with $70 \mu \mathrm{L}$ of $200 \mathrm{mmol} / \mathrm{L}$ sodium bicarbonate solution. The absorbance and fluorescence spectra of the QD sample were immediately recorded after neutralizing the sample. The observed spectral changes in the neutralized solutions were similar to those in the corresponding original etching solutions. To obtain the $t=0$ data point, $70 \mu \mathrm{L}$ of $200 \mathrm{mmol} / \mathrm{L}$ sodium bicarbonate solution was added to the warm SGF followed by the addition of QDs; the absorbance and fluorescence spectra were immediately obtained. Control studies were performed by incubating each QD type in PBS at $37^{\circ} \mathrm{C}$.

Cytotoxicity studies. Caco-2 cells were purchased from ATCC (Manassas, VA). The cells were cultured in Eagle's minimum essential medium (MEM; Mediatech Inc., Herndon, VA) that contained glucose $(1 \mathrm{~g} / \mathrm{L})$, L-glutamine $(2 \mathrm{mmol} / \mathrm{L})$, and $10 \%$ fetal bovine serum (FBS). The cells were cultured and passaged according to instructions from ATCC (www.atcc.org). The cytotoxicity of protonrepelling QDs was assessed by quantifying damage to the cell membranes. First, Caco-2 cells in their logarithmic growth phase were seeded on a blacksided, clear bottom 96-well assay plate at a density of $3.2 \times 10^{4}$ cells / well and allowed to form a confluent monolayer, over approximately $48 \mathrm{~h}$. The media was removed and the cells were washed with PBS. Next, $50 \mu \mathrm{L}$ of complete media followed by $50 \mu \mathrm{L}$ of complete media containing QDs at concentrations of 2,10 , or $20 \mathrm{nmol} / \mathrm{L}$ was added to the cells. Triton $\mathrm{X}-100$ and complete media were used as positive and negative controls, respectively. After $2 \mathrm{~h}$, cell membrane damage was calculated by measuring lactate dehydrogenase (LDH) release into cell culture media using the Cytotox-ONE ${ }^{\mathrm{TM}}$ Homogeneous Membrane Integrity Assay (Promega Corp., Madison, WI). The assay was performed according to the manufacturer's instructions. Briefly, a cocktail of lactate, $\mathrm{NAD}^{+}$, resazurin, and diaphorase was added to the cell culture media. The LDH, which is only released from cells with a damaged membrane, reacts with lactate and $\mathrm{NAD}^{+}$to produce NADH and pyruvate. The NADH then reacts with resazurin in the presence of diaphorase to produce highly fluorescent resorufin in the enzyme-coupled reaction. The fluorescence intensity in each well from resorufin was measured using a BioTek Synergy-2 (Winooski, VT) microplate reader, equipped with $530 / 25 \mathrm{~nm}$ and 590/35 nm excitation and emission filters. Cytotoxicity, as measured by cell membrane damage, was normalized to the negative media control and the Triton X-100 positive control. 


\section{Results}

As depicted schematically in Fig. 1(a), a hydrophobic layer of an amphiphilic polymer or lipid coating on QDs is ineffective in blocking protons from reaching the QD surface and acid etching leads to lattice defects on the QD surface; these defects are known to cause fluorescence quenching [10]. Protonsponge polymers are good candidates as protonresistant surface coatings because, due to electrostatic repulsion, the positively charged coatings should prevent free protons from reaching the QD surface, thus protecting QDs from acid etching, as depicted schematically in Fig. 1(b). The changes in optical absorbance and fluorescence of lipid-PEGcoated QDs with time of immersion in an acidic environment simulating gastric fluids are shown in Fig. 2. The absorbance spectra of these QDs show a definite blue-shift from $610 \mathrm{~nm}$ to $604 \mathrm{~nm}$ in the first exciton peak, but the magnitude of absorption (optical density) stays nearly constant. In comparison, the fluorescence emission spectra show both a blueshift in wavelength and a dramatic drop in intensity; the fluorescence is quenched by more than $99 \%$ after $60 \mathrm{~min}$. These observations demonstrate that the observed fluorescence quenching of lipidPEG-encapsulated QDs is caused by acid-induced etching, and not by aggregation or precipitation. This finding is consistent with that of Smith et al. [11], who showed that lipid-PEG-encapsulated QDs are chemically etched at $\mathrm{pH} 1-2$. In contrast, in the nearly neutral environment of simulated intestinal fluids (pH 6.5 and $10 \mathrm{mg} / \mathrm{mL}$ pancreatin) or neutral PBS at $37^{\circ} \mathrm{C}$, the fluorescence of lipid-PEG-encapsulated QDs is essentially unchanged after $3 \mathrm{~h}$.

Recent work [19] has shown that the use of PEI and PEI-g-PEG coatings can improve the acid stability of QDs. We have therefore examined the ability of these coatings to prevent loss of QD fluorescence on immersion in SGF. As shown in Fig. 3, the PEI-coated QDs retained over $50 \%$ of their fluorescence intensity after $1 \mathrm{~h}$ of exposure, while the PEI- $g$-PEG-coated QDs retained approximately $70 \%$ of their initial fluorescence intensity. Both of these QDs have significantly more stable fluorescence emission when compared to lipid-PEG-coated QDs.
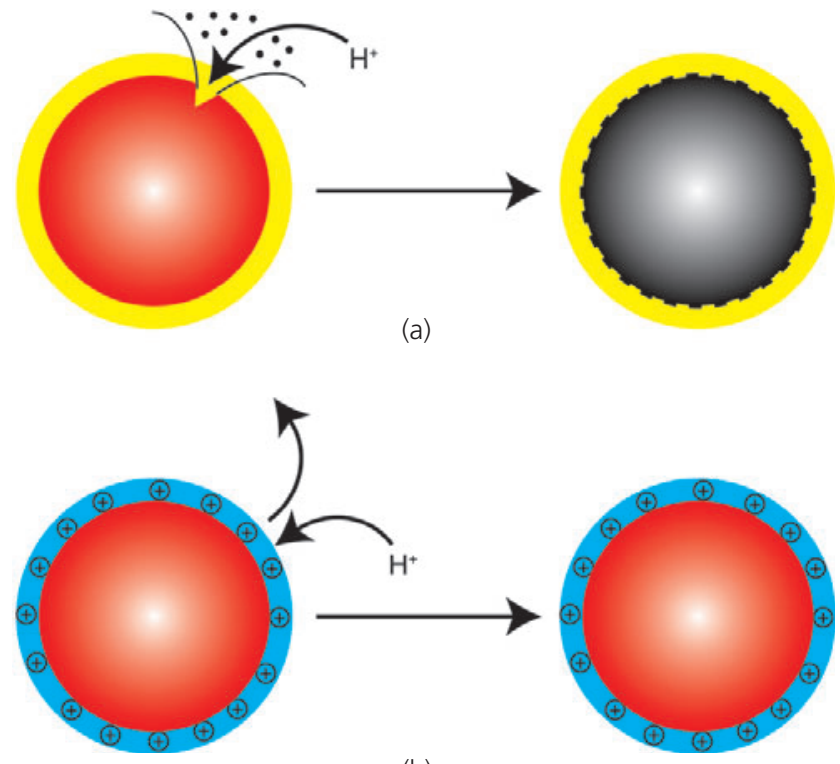

(b)

Figure 1 Schematic diagrams of (a) acid-etchable and (b) protonresistant QDs. Acid etching leads to surface defects and fluorescence quenching, as observed for QDs coated with amphiphilic polymers or lipids. The use of cationic "proton-resistant" surface coatings prevents free protons from reaching the nanocrystal surface, thus protecting QDs from acid-induced etching

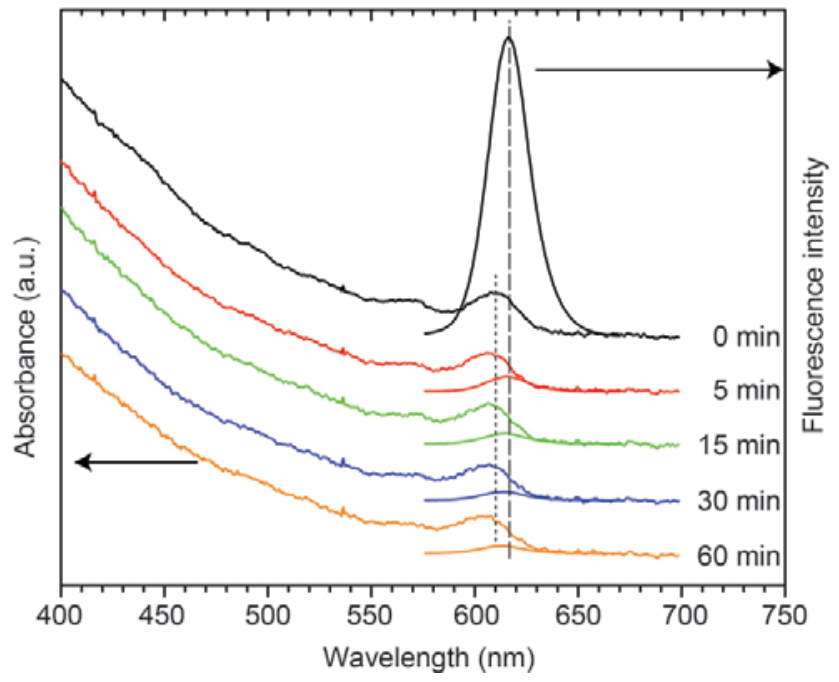

Figure 2 Optical absorbance and fluorescence spectral changes showing acid-induced etching of lipid-encapsulated QDs in simulated gastric fluids ( $\mathrm{pH}$ 2). Note the blue shift in the absorbance peak (short dashes) and quenching of the fluorescence signal (long dashes) with increasing exposure time to the acidic solution

A potential disadvantage of the PEI-coated and PEI- $g$-PEG QDs is that they are prepared by ligand exchange reactions [19], and are less colloidally stable than lipid-PEG or amphiphilic polymer-coated QDs. 
When stored at $4{ }^{\circ} \mathrm{C}$ in aqueous solution, however, we found that the PEI-coated dots maintained over $40 \%$ of their original fluorescence intensity after one year.

The cationic surfaces employed in this work have a large buffering capacity, which likely contributes to the acid stability. The zeta potentials for PEI and PEI-g-PEG-coated QDs at neutral $\mathrm{pH}$ are $+29 \mathrm{mV}$ and $+22 \mathrm{mV}$, respectively. In SGF $(\mathrm{pH} 2)$, these surfaces are expected to have more positive charges and higher zeta potentials. Thus,

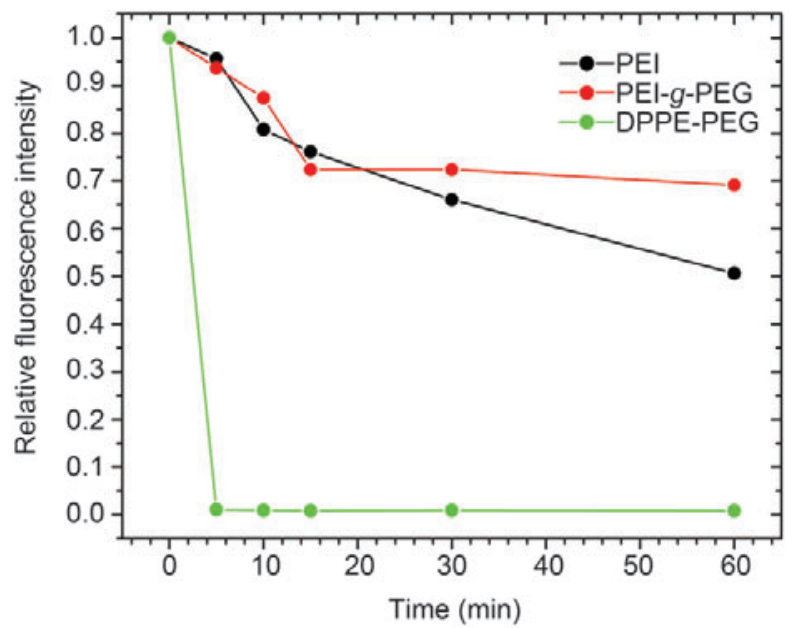

(a)

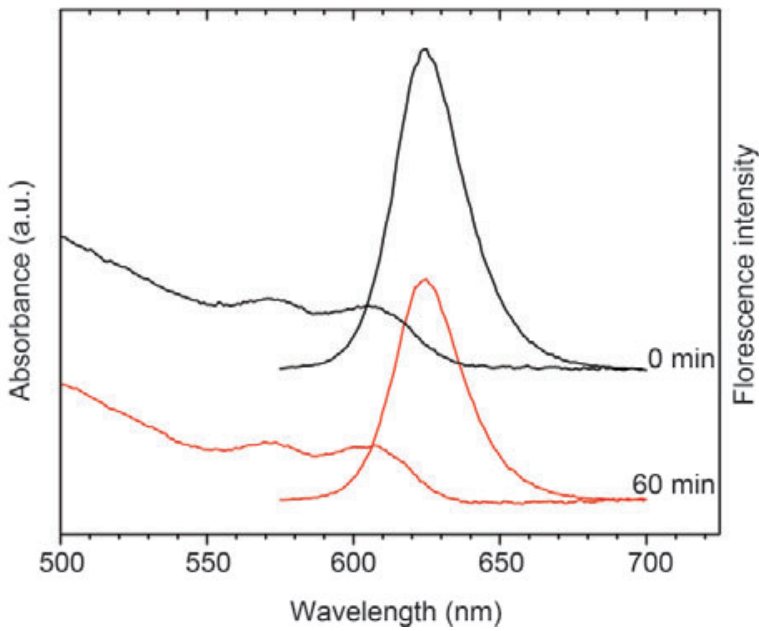

(b)

Figure 3 (a) Comparison of fluorescence signals between traditional amphiphilic PEG-coated QDs and proton-resistant QDs in simulated gastric fluids. Under similar acidic conditions, QDs coated with traditional amphiphilic polymers (octylamine-modified polyacrylic acid) are both quenched and precipitated; (b) absorbance and fluorescence spectra of proton-resistant QDs at different etching times. Notice that there is no blue-shift for either absorbance or fluorescence and that > $50 \%$ of the fluorescence intensity remains after $60 \mathrm{~min}$ we believe that free protons in gastric fluids are unable to penetrate the protective cationic shells of PEI and PEI-g-PEG-coated QDs. In essence, these "acid-resistant" coatings create a "proton buffer" guarding the QD core-shell structure from highly destructive protons. Figure 4 qualitatively shows a direct comparison between acid-etchable QDs and acid-resistant QDs that have been treated with SGF. The PEI-coated QDs are brightly fluorescent even after $60 \mathrm{~min}$, whereas the lipid-PEG-coated QDs are largely quenched.

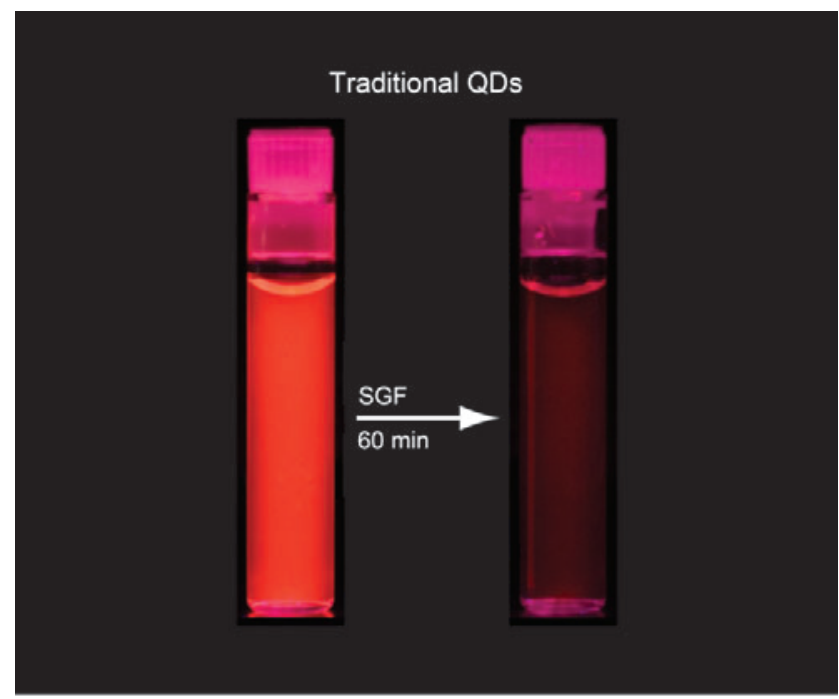

(a)

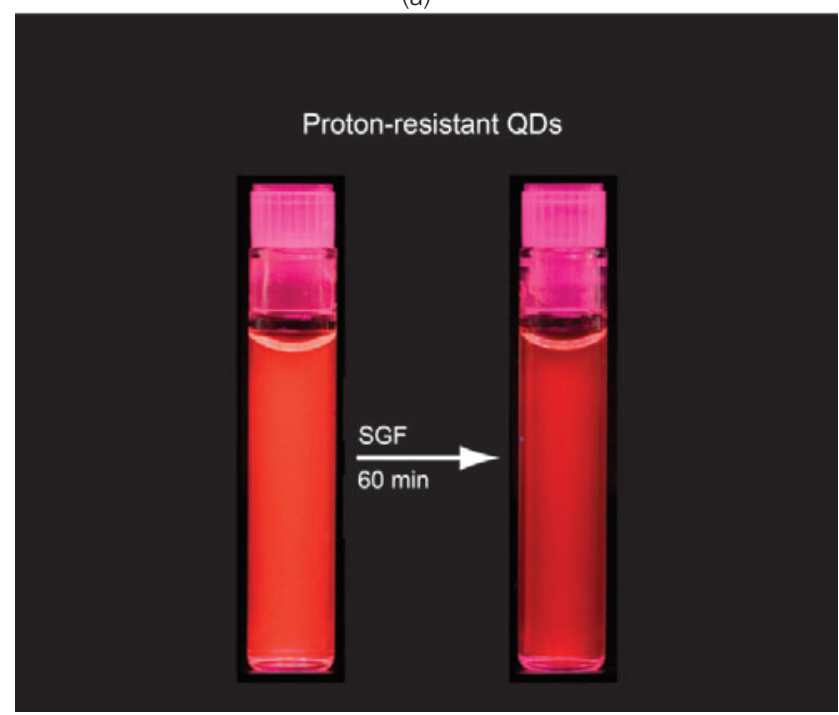

(b)

Figure 4 Visualization of (a) traditional and (b) proton-resistant quantum dots before and after acid etching in SGF for $60 \mathrm{~min}$. The initial fluorescence images were obtained by neutralizing SGF with sodium bicarbonate and then adding QD solution, while the etched QD fluorescence images were obtained by incubating QDs in SGF for $60 \min$ at $37^{\circ} \mathrm{C}$ 
These acid-stable QDs are likely to find use in oral delivery applications because they can retain their fluorescence throughout the gastrointestinal tract and can be detected by sensitive fluorescence imaging. Toward this goal, we have examined the biocompatibility of these QDs by using Caco-2 cells, a type of epithelial colorectal adenocarcinoma that is widely used to predict the absorption rate of candidate drug compounds across the intestinal epithelial cell barrier [18]. Because the proton-resistant QDs are positively charged, their cellular toxicity is most likely to arise from cell membrane disruption and other damage [23]. Accordingly, we used an in vitro toxicity assay to measure membrane damage and the subsequent release of LDH. Figure 5 shows the membrane damage of Caco-2 cells upon exposure to acidstable QDs. At a concentration of $1 \mathrm{nmol} / \mathrm{L}$, both cationic polymer-coated QDs are found to damage the cell membrane, resulting in a higher LDH release after $2 \mathrm{~h}$. The PEI-coated QDs are more toxic than PEI-g-PEG-coated QDs, but both are significantly less toxic than Triton X-100 (used here as a positive control). Overall, the cytotoxicity results underscore the need to improve the biocompatibility of proton-resistant QDs, perhaps by PEG grafting or other chemical modifications.

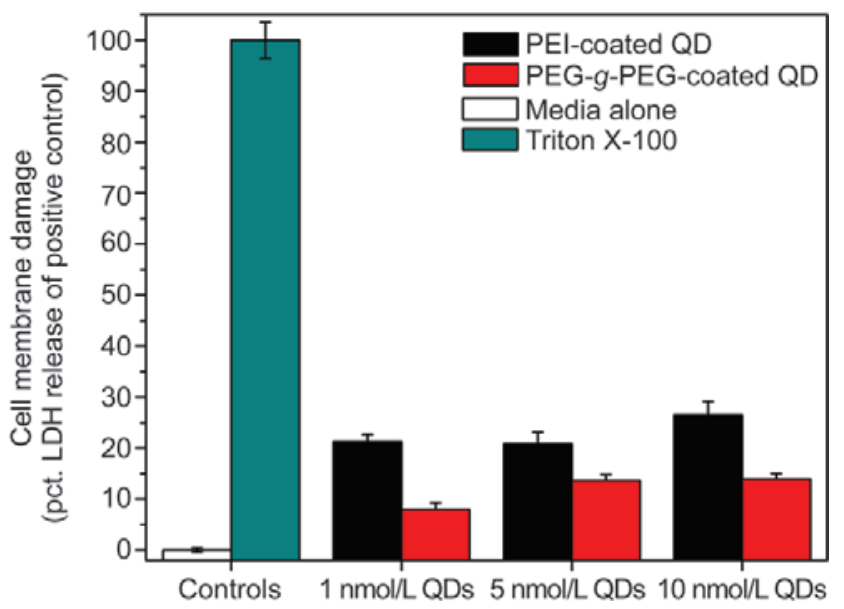

Figure 5 Cellular cytotoxicity of proton-resistant QDs measured by $L D H$ release in Caco-2 cells as a result of membrane damage. Triton X-100, a cell permeabilizing agent, was used as the positive control and represented the maximum possible LDH release $(100 \%$ LDH). The cell culture media was used as the negative control $(0 \%$ $\mathrm{LDH}$ release)

\section{Discussion}

A number of procedures have been used to encapsulate and solubilize hydrophobic semiconductor QDs for biological applications. Most of these procedures are based on the use of small-molecule coordinating ligands, amphiphilic polymers, or amphiphilic lipids [4-9]. A recent systematic study by Smith et al. [11] has shown that the surface coating chemistry has a dramatic effect on the hydrodynamic size, fluorescence quantum yield, photostability, chemical stability, and biocompatibility of water-soluble QDs. Quantum dots with the smallest hydrodynamic sizes are best prepared by ligand exchange with hydrophilic molecules, but the resulting particles are less stable than those encapsulated in amphiphilic polymers. For stability against chemical oxidation, QDs should be protected with a hydrophobic bilayer. For stability in high salt buffers, it is preferable to have uncharged, sterically-stabilized QDs, like those coated with PEG. For high stability under acidic conditions, our results show that QDs should be protected with a proton-sponge polymer layer such as branched PEI. Gao and co-workers have also prepared QDs coated with a proton-sponge having a balanced mixture of tertiary amines and carboxylic acid groups, and have demonstrated that this class of QDs can be used for efficient small interfering RNA (siRNA) delivery and real-time intracellular imaging [17]. The protonsponge QDs are "endosomolytic" because they are able to absorb a large number of protons in acidic organelles and can cause endosomal rupture when a large osmotic pressure imbalance is built up across the organelle membrane [16].

The development of proton-resistant surface coatings also opens new opportunities for directly observing and studying QDs in harsh physiological environments. In particular, acid-stable QDs could be used as model probes to predict the oral absorption and biodistribution of therapeutic nanoparticles. Fluorescence emission of acid-stable QDs would be relatively stable in the gastrointestinal tract, specifically in the stomach, where the $\mathrm{pH}$ can be as low as 1-2 [15]. Therefore, the uptake and biodistribution of orally administered nanoparticles 
could be tracked in real time.Therapeutic delivery via the oral route is the preferred method for drug administration because it is convenient and avoids painful, repeated injections. Oral delivery of many pharmaceuticals, including anticancer cytotoxic agents, is limited because of their low bioavailability $[24,25]$. The poor bioavailability of orally delivered therapeutics is due to their degradation in the highly acidic and enzyme-rich gastric fluids, especially for bioactive pharmaceuticals [26, 27], and their poor intestinal absorption [24, 28]. Polymers sensitive to $\mathrm{pH}$ changes, such as enteric methacrylate coatings, have been used to improve the gastric stability of therapeutics because these polymers become hydrophobic at low $\mathrm{pH}$ and entrap the desired therapeutic compound [29-32]. Above a defined dissolution $\mathrm{pH}$, usually between $\mathrm{pH} 4$ and 7 , the polymer ionizes and dissolves releasing free drug in the large or small intestine. Although this decreases degradation of the drug in gastric fluids, the drug must still be absorbed through the intestinal barrier and survive first-pass liver metabolism. Nanoparticle therapeutics offer a promising benefit to oral delivery because they can be internalized by cells through pinocytosis or by adsorptive/receptor-mediated endocytosis, thereby avoiding the P-glycoprotein efflux pumps and promote systemic circulation [33-36]. In addition to transcytosis, the size of nanoparticles renders them ideal particles to exploit the highly phagocytic M-cells of gut-associated lymphatic tissue (GALT) for potential vaccine delivery [21, 37-40].

The delivery of nanoparticles can be monitored in vivo and in vivo by both qualitative imaging methods such as electron and fluorescence microscopy [4042], and by quantitative chemical and biochemical methods such as spectrophotometry, liquid chromatography, elemental analysis, immunoassays, and reverse transcriptase polymerase chain reactions (RT-PCR) [21, 32, 35, 40, 43]. Chemical methods and therapeutic response studies give important quantitative endpoint information, but do not address the biodistribution mechanism. Electron microscopy (EM) provides detailed structural information, but cannot provide real time information on nanoparticle biodistribution nor can it provide reliable data for nanoparticles that are not electron dense. Experiments implementing fluorescence imaging and microscopy techniques often utilize nanoparticles that are loaded with small organic dyes. Since these dyes can be chemically cleaved or leached from the nanoparticle, they may not reliably report the location or concentration of the nanoparticles. In this work we have reported early results on two acid-stable QDs in simulated gastrointestinal fluids, and these studies are an important step towards the development of nanoparticle agents that can effectively cross the gastrointestinal tract for imaging and therapeutic purposes.

\section{Conclusions}

We have reported the development of protonresistant QDs based on the use of proton-sponge polymer coatings for potential applications in harsh physiological conditions, especially the highly acidic gastric environment. In contrast to the rapid fluorescence quenching of traditional QDs in acid, both PEI and PEI-g-PEG-coated QDs show excellent fluorescence stability in simulated gastric fluids. This effect is believed to arise from a proton buffering layer that prevents free protons from reaching the QD surface, thus protecting QDs from acid-induced etching. Due to their colloidal and fluorescence stability in highly degradative gastric fluids, protonresistant QDs have promise as a new class of nanoparticles for oral delivery applications. Future work will need to minimize the cellular toxicity of proton-resistant QDs.

\section{Acknowledgements}

The work was partly supported by NIH Grants (P20 GM072069, R01 CA108468-01, U01 HL080711, and U54CA119338) and the Georgia Cancer Coalition Distinguished Cancer Scholars Program (to S. N.).

\section{References}

[1] Alivisatos, P. The use of nanocrystals in biological detection. Nat. Biotech. 2004, 22, 47-52.

[2] Michalet, X.; Pinaud, F. F.; Bentolila, L. A.; Tsay, J. M.; 
Doose, S.; Li, J. J.; Sundaresan, G.; Wu, A. M.; Gambhir, S. S.; Weiss, S. Quantum dots for live cells, in vivo imaging, and diagnostics. Science 2005, 307, 538-544.

[3] Gao, X.; Yang, L.; Petros, J. A.; Marshall, F. F.; Simons, J. W.; Nie, S. In vivo molecular and cellular imaging with quantum dots. Curr. Opin. Biotechnol. 2005, 16, 63-72.

[4] Smith, A. M.; Duan, H.; Mohs, A. M.; Nie, S. Bioconjugated quantum dots for in vivo molecular and cellular imaging. Adv. Drug Deliv. Rev. 2008, 60, 12261240.

[5] Wu, X.; Liu, H.; Liu, J.; Haley, K. N.; Treadway, J. A.; Larson, J. P.; Ge, N.; Peale, F.; Bruchez, M. P. Immunofluorescent labeling of cancer marker Her2 and other cellular targets with semiconductor quantum dots. Nat. Biotechnol. 2003, 21, 41-46.

[6] Gao, X. H.; Cui, Y. Y.; Levenson, R. M.; Chung, L. W. K.; Nie, S. M. In vivo cancer targeting and imaging with semiconductor quantum dots. Nat. Biotechnol. 2004, 22, 969-976.

[7] Dubertret, B.; Skourides, P.; Norris, D. J.; Noireaux, V.; Brivanlou, A. H.; Libchaber, A. In vivo imaging of quantum dots encapsulated in phospholipid micelles. Science 2002, 298, 1759-1762.

[8] Smith, A. M.; Nie, S. Minimizing the hydrodynamic size of quantum dots with multifunctional multidentate polymer ligands. J. Am. Chem. Soc. 2008, 130, 11278-11279.

[9] Kairdolf, B. A.; Smith, A. M.; Nie, S. One-pot synthesis, encapsulation, and solubilization of size-tuned quantum dots with amphiphilic multidentate ligands. J. Am. Chem. Soc. 2008, 130, 12866-12867.

[10] Mancini, M. C.; Kairdolf, B. A.; Smith, A. M.; Nie, S. Oxidative quenching and degradation of polymerencapsulated quantum dots: New insights into the longterm fate and toxicity of nanocrystals in vivo. J. Am. Chem. Soc. 2008, 130, 10836-10837.

[11] Smith, A. M.; Duan, H. W.; Rhyner, M. N.; Ruan, G.; Nie, S. M. A systematic examination of surface coatings on the optical and chemical properties of semiconductor quantum dots. Phys. Chem. Chem. Phys. 2006, 8, 38953903.

[12] Winterbourn, C. C. Reconciling the chemistry and biology of reactive oxygen species. Nat. Chem. Biol. 2008, 4, 278-286.

[13] Fruehauf, J. P.; Meyskens, F. L. Jr. Reactive oxygen species: A breath of life or death. Clin. Cancer Res. 2007, 13, 789-794.
[14] Leopold, J. A.; Loscalzo, J. Oxidative enzymopathies and vascular disease. Arterioscler. Thromb. Vasc. Biol. 2005, 25, 1332-1340.

[15] Lindahl, A.; Ungell, A. L.; Knutson, L.; Lennernas, H. Characterization of fluids from the stomach and proximal jejunum in men and women. Pharm. Res. 1997, 14, 497 -502 .

[16] Boussif, O.; Lezoualc'h, F.; Zanta, M. A.; Mergny, M. D.; Scherman, D.; Demeneix, B.; Behr, J. P. A versatile vector for gene and oligonucleotide transfer into cells in culture and in vivo: Polyethylenimine. Proc. Natl. Acad. Sci. USA 1995, 92, 7297-7301.

[17] Yezhelyev, M. V.; Qi, L.; O'Regan, R. M.; Nie, S.; Gao, X. Proton-sponge coated quantum dots for siRNA delivery and intracellular imaging. J. Am. Chem. Soc. 2008, 130, 9006-9012.

[18] Artursson, P.; Palm, K.; Luthman, K. Caco-2 monolayers in experimental and theoretical predictions of drug transport. Adv. Drug Deliv. Rev. 2001, 46, 27-43.

[19] Duan, H.; Nie, S. Cell-penetrating quantum dots based on multivalent and endosome-disrupting surface coatings. J. Am. Chem. Soc. 2007, 129, 3333-3338.

[20] Qu, L. H.; Peng, X. G. Control of photoluminescence properties of CdSe nanocrystals in growth. J. Am. Chem. Soc. 2002, 124, 2049-2055.

[21] Rae, C. S.; Khor, I. W.; Wang, Q.; Destito, G.; Gonzalez, M. J.; Singh, P.; Thomas, D. M.; Estrada, M. N.; Powell, E.; Finn, M. G.; Manchester, M. Systemic trafficking of plant virus nanoparticles in mice via the oral route. Virology 2005, 343, 224-235.

[22] Takagi, K.; Teshima, R.; Okunuki, H.; Sawada, J. Comparative study of in vitro digestibility of food proteins and effect of preheating on the digestion. Biol. Pharm. Bull. 2003, 26, 969-973.

[23] El-Sayed, M.; Ginski, M.; Rhodes, C.; Ghandehari, $\mathrm{H}$. Transepithelial transport of poly (amidoamine) dendrimers across Caco-2 cell monolayers. J. Control. Release 2002, 81, 355-365.

[24] Sparreboom, A.; van Asperen, J.; Mayer, U.; Schinkel, A. H.; Smit, J. W.; Meijer, D. K. F.; Borst, P.; Nooijen, W. J.; Beijnen, J. H.; van Tellingen, O. Limited oral bioavailability and active epithelial excretion of paclitaxel (Taxol) caused by $P$-glycoprotein in the intestine. Proc. Natl. Acad. Sci. U. S. A. 1997, 94, 2031-2035.

[25] Schellens, J. H. M.; Malingré, M. M.; Kruijtzer, C. M. F.; Bardelmeijer, H. A.; van Tellingen, O.; Schinkel, A. 
$\mathrm{H}$.; Beijnen, J. H. Modulation of oral bioavailability of anticancer drugs: From mouse to man. Eur. J. Pharm. Sci. 2000, 12, 103-110.

[26] Goldberg, M.; Gomez-Orellana, I. Challenges for the oral delivery of macromolecules. Nat. Rev. Drug Discov. 2003, 2, 289-295.

[27] Woodley, J. F. Enzymatic barriers for Gl peptide and protein delivery. Crit. Rev. Ther. Drug Carrier Syst. 1994, $11,61-95$.

[28] Malingre, M. M.; Beijnen, J. H.; Schellens, J. H. Oral delivery of taxanes. Invest. New Drugs 2001, 19, 155162.

[29] Bromberg, L. Intelligent polyelectrolytes and gels in oral drug delivery. Curr. Pharm. Biotechnol. 2003, 4, 339349.

[30] De Jaeghere, F.; Allemann, E.; Kubel, F.; Galli, B.; Cozens, R.; Doelker, E.; Gurny, R. Oral bioavailability of a poorly water soluble HIV-1 protease inhibitor incorporated into $\mathrm{pH}$-sensitive particles: Effect of the particle size and nutritional state. J. Control. Release 2000, 68, 291-298.

[31] Li, M. G.; Lu, W. L.; Wang, J. C.; Zhang, X.; Zhang, H.; Wang, X. Q.; Wu, C. S.; Zhang, Q. Preparation and characterization of insulin nanoparticles employing chitosan and poly(methylmethacrylate / methylmethacrylic acid) copolymer. J. Nanosci. Nanotechnol. 2006, 6, 2874 -2886 .

[32] Sarmento, B.; Ribeiro, A.; Veiga, F.; Ferreira, D.; Neufeld, R. Oral bioavailability of insulin contained in polysaccharide nanoparticles. Biomacromolecules 2007, 8, 3054-3060.

[33] Ambudkar, S. V.; Dey, S.; Hrycyna, C. A.; Ramachandra, M.; Pastan, I.; Gottesman, M. M. Biochemical, cellular, and pharmacological aspects of the multidrug transporter. Annu. Rev. Pharmacol. Toxicol. 1999, 39, 361-398.

[34] Gottesman, M. M.; Fojo, T.; Bates, S. E. Multidrug resistance in cancer: Role of ATP-dependent transporters.
Nat. Rev. Cancer 2002, 2, 48-58.

[35] Roy, K.; Mao, H. Q.; Huang, S. K.; Leong, K. W. Oral gene delivery with chitosan-DNA nanoparticles generates immunologic protection in a murine model of peanut allergy. Nat. Med. 1999, 5, 387-391.

[36] Win, K. Y.; Feng, S. S. Effects of particle size and surface coating on cellular uptake of polymeric nanoparticles for oral delivery of anticancer drugs. Biomaterials 2005, 26, 2713-2722.

[37] Beier, R.; Gebert, A. Kinetics of particle uptake in the domes of Peyer's patches. Am. J. Physiol. 1998, 275, G130-137.

[38] Neutra, M. R.; Mantis, N. J.; Frey, A.; Giannasca, P. J. The composition and function of $\mathrm{M}$ cell apical membranes: Implications for microbial pathogenesis. Semin. Immunol. 1999, 11, 171-181.

[39] des Rieux, A.; Fievez, V.; Garinot, M.; Schneider, Y. J.; Preat, $V$. Nanoparticles as potential oral delivery systems of proteins and vaccines: A mechanistic approach. J. Control. Release 2006, 116, 1-27.

[40] Hillyer, J. F.; Albrecht, R. M. Gastrointestinal persorption and tissue distribution of differently sized colloidal gold nanoparticles. J. Pharm. Sci. 2001, 90, 1927-1936.

[41] Behrens, I.; Pena, A. I. V.; Alonso, M. J.; Kissel, T. Comparative uptake studies of bioadhesive and nonbioadhesive nanoparticles in human intestinal cell lines and rats: The effect of mucus on particle adsorption and transport. Pharm. Res. 2002, 19, 1185-1193.

[42] Prego, C.; Fabre, M.; Torres, D.; Alonso, M. J. Efficacy and mechanism of action of chitosan nanocapsules for oral peptide delivery. Pharm. Res. 2006, 23, 549-556.

[43] Sonavane, G.; Tomoda, K.; Sano, A.; Ohshima, H.; Terada, H.; Makino, K. In vitro permeation of gold nanoparticles through rat skin and rat intestine: Effect of particle size. Colloids Surf. B Biointerfaces 2008, 65, 110. 